\title{
XXI.
}

Aus der Heidelberger medicinischen Klinik.

\section{Ueber Athetose als Complication von Tabes dorsalis.}

\author{
Von \\ Dr. Arnsperger, \\ Volontärarzt der Klinik.
}

Das ungemein mannigfaltige, durch die Fülle von Symptomen ausgezeichnete Krankheitsbild der Tabes dorsalis wird häufig noch durch das Hinzutreten von Erscheinungen complicirt, welche nicht zum eigentlichen Symptomencomplex gehören und deren Zusammenhang oder Abhängigkeit von der Tabes noch unbekannt oder umstritten ist.

Eine sehr seltene, aber in mancher Hinsicht grosses Interesse darbietende Complication der Tabes ist die Athetose, und es dürfte deshalb Berechtigung haben, zwei Fälle dieser Art, wenn sie auch nur klinisch beobachtet sind, mitzutheilen.

Bei dem ersten Falle, welcher anf der hiesigen Klinik beobachtet wurde, handelt es sich um einen 43 jährigen, ledigen Schmied H. E.

Der Pat. hatte im Jahre 1880 ein Ulcus durum der Glans penis, in der darauf folgenden Zeit Drüsenschwellungen, Ausschlag und andere Secundärerscheinungen. Er wurde nicht mit specifischen Mitteln behandelt und erst im Jahre 1897 fing er eine Inunctionseur an, welche aber schon nach 8 Einreibungen beendet wurde. Darnach nahm Pat. noch längere Zeit Jodkalium.

Seit dem Jahre 1896 waren schon Erscheinungen einer Tabes anfgetreten; zunächst Parästhesien in Fingern und Zehen, dann 1897 Unsicherheit des Gehens, lancinirende Schmerzen, 1898 Abnahme des Sehvermögens, Ende 1899 Unsicherheit im Dunkeln, Gürtelgefühl und Abnahme der Potenz.

Bei der Aufnahme am 23. März 1900 bot der Kranke das typische Bild der Tabes dorsalis dar.

Die Symptome waren: Ataxie, reflectorische Pupillenstarre, Schwanken beim Schliessen der Angen, Fehlen der Sehnenreflexe, Sensibilitätsstörungen an den Handflächen und Fusssohlen, Störungen der Muskel- und Gelenksensibilität, Hypotonie, Ulnaris-Analgesie (Bi erna cki), Hodenanalgesie, Kältehyperästhesie am Rumpfe, Atrophie der Papillen mit leichter Gesichtsfeldeinschränkung, leichte Sphincterenstörungen, dazu subj. Gürtelgefühl, lancinirende Schmerzen, Ulnaris-Parästhesien. 
Bot der Fall somit das klassische Bild der Tabes dorsalis, so fielen als etwas nicht dazu Gehöriges sofort langsame, ständig sich wiederholende Bewegungen der Finger beider Hände auf, welche die typischen Zeichen athetotischer Bewegungen, wie sie Hammond 1871 zuerst als besonderen Symptomencomplex beschrieben hat, aufwiesen.

Die Bewegungen bestanden in wurmförmig-kriechenden, langsamen, continuirlichen Streckungen und Beugungen der Finger, welche bis zu übertriebenen Streck- und Beugestellungen führten, dann wieder Spreizung der Finger und Zusammenziehen in Schreibstellung.

Gerade die ausserordentliche Langsamkeit und Trägheit, sowie die Uebertreibung normaler Bewegungen and die Bildung complicirter Fingerstellungen fielen besonders auf.

Auch in den Beinen konnten derartige athetotische Bewegungen beobachtet werden, welche sich aber hier nur auf die Adduction der Oberschenkel und die Einwärtsrotation des Beines beschränkten.

Im Schlafe hörten die Athetosebewegungen auf, bei psychischen Erregungen, oder körperlichen Anstrengungen konnte ein Stärkerwerden der Bewegungen beobachtet werden.

Willkürlich sind die Bewegungen wohl etwas zu hemmen; sobald aber die Aufmerksamkeit abgelenkt wird, treten sie wieder auf.

Der Kranke hatte von dem Bestehen dieser Athetose keine Ahnung; er kann deshalb natïrlich nicht angeben, seit wann dieselben etwa bestehen; ob er dieselben erst im Verlanf seiner Krankheit bekommen hat, oder ob dieselben schon früher, vielleicht schon von Jugend auf bestanden haben. Immerhin ist es, gerade deshalb weil der Kranke nichts von der Störung weiss, wahrscheinlicher, dass dieselbe erst im Verlauf der Erkrankung eingetreten ist, als die Gebrauchsfähigkeit der Hände durch die Ataxie schon erheblich gelitten hatte.

Die Athetosebewegungen blieben auch im weiteren Verlaufe unserer Beobachtung bestehen und besserten sich in keiner Weise, obwohl das Grundleiden auf die specifische Behandlung hin entschieden Besserung erkennen liess, und gerade die andere, die intendirte Motilitätsstörung, die Ataxie, durch länger fortgesetzte Uebungstherapie bedeutende Fortschritte in der Besserung machte.

Noch eine zweite, nur selten beobachtete Anomalie liess sich bei diesem Falle finden, es war dies das Symptom der intermittirenden Pupillenstarre: Erb hat schon darauf hingewiesen, dass das Argyll Robertson'sche Symptom, wenn es einmal zu Stande gekommen ist, nicht constant bestehen bleiben muss; anch v. Leyden und Goldscheider erwähnen die Schwankungen, welche das Symptom aufweisen kann. Eichhorst ${ }^{1}$ ) hat unter 103 Tabesfällen 2 mal diese Schwankungen beobachten und verfolgen können.

Bei unserem Kranken konnten Schwankungen in der Pupillenreaction mehrmals während der fast 2 monatlichen Beobachtungszeit constatirt werden, wobei natürlich die Untersuchungsbedingungen völlig gleichmässig und einwandsfrei angeordnet waren, da man bei der Prüfung der Reaction der Papillen auf Lichteinfall leicht Täuschungen ausgesetzt ist.

1) Eichhorst, Einige Bemerkungen über intermittirende Pupillenstarre bei Tabes dorsalis. Deutsche med. Wochenschrift 1898. Nr. 23. 
Der zweite Fall von Combination der Tabes dorsalis mit Athetose bot ein ähnliches Bild.

Auch hier war der ausgesprochene Symptomencomplex der Tabes bei einem 51jährigen Taglöhner $\mathrm{C}$. W., bestehend in Ataxie, Trägheit der Pupillarreaction, Ungleichheit der Pupillen, Schwanken beim Schliessen der Angen, Störungen der Muskel- und Gelenksensibilität, leichten Tastempfindungsstörungen der Fusssohlen, Parästhesien, lancinirenden Schmerzen, Fehlen der Sehnenreflexe, Sphincterenstörungen, zu constatiren.

Allerdings bestand neben diesen Symptomen noch Nystagmus, welcher wie bekannt ja nicht zum Bilde der 'Tabes gehört; dieser Nystagmus verschwand übrigens zeitweise auch wieder, so dass wir offenbar nicht den typischen Nystagmus vor uns hatten. Zeitweise bestand daneben auch noch eine linksseitige Abducensparese.

Die Athetosebewegungen sind sowohl an den Händen, als auch an den Füssen und auch an der Zunge beobachtet und gleichen genan den Bewegungen, wie sie der vorher beschriebene Fall aufwies.

Neben den langsamen, wurmförmigen, ständig sich abspielenden Bewegungen der Finger, Zehen und Zunge sind aber an dem Kranken noch andere unwillkürliche Bewegungen zu beobachten, unwillkürliche, kurze Zuckungen in den Beinen, unwillkürliche Adductorencontractionen der Oberschenkel, wie sie bei Tabes nicht selten gefunden werden, welche aber scharf zu unterscheiden sind von der Athetose.

Wenn es sich bei diesen Zuckungen, wie sie neuerdings Hirschberg 1) in der Raymond'schen Klinik an grossem Material studirt hat, auch um unwillkürliche, spontan auftretende Bewegungen handelt, so sind sie viel zu unregelmässig, zu kurz, zu wenig complicirt, um nur irgend mit den langsamen, regelmässigen und complicirten Athetosebewegungen zusammengestellt werden zu können.

Die Ruhelosigkeit der befallenen Glieder, die Uebertreibung der Extension und Flexion, Adduction und Abduction, welche bis zu Veränderungen im Bandappart und bis zur Deformirung der Gelenke selbst führt, die Steigerung der Bewegungen bei psychischen Erregungen, das Sistiren im Scblafe sind die Charakteristica der Athetosebewegungen.

Auch die mehrmals als Combination von Tabes dorsalis beschriebenen choreaartigen Motilitätsstörungen sind wohl zum grossen Theil von den irregulären Zuckungen scharf zu trennen und häufig eher der Gruppe der athetotischen Bewegung zuzurechnen; doch ist bei diesen choreiformen Bewegungen eine ebenso scharfe Abtrennung, wie es bei der Athetose der Fall ist, nicht immer möglich.

Bevor ich nun auf die wichtige Frage der Localisation der Athetose,

1) Hirschberg, Des mouvements involontaires spontanés chez les tabétiques. Revue neurolog. 1897. Ref. Arch. de neurol. 1898. 
auf die Besprechung des Zusammenhanges der beiden combinirten Erkrankungen Tabes und Athetose eingehe, möchte ich die wenigen in der Literatur niedergelegten Fälle der gleichen Combination berühren.

Rosenbach ${ }^{1}$ ) hat zuerst einen derartigen Fall publicirt; doch ist aus der Beschreibung zu entnehmen, dass die Motilitätsstörung nicht rein athetotischer Natur war, sondern dass auch choreiforme Bewegungen beigemischt waren.

Bemerkenswerth ist der Fall dadurch, dass es der einzige ist, welcher zur Obduction gekommen ist. Bei der Section fand sich nun im Rückenmark das typische Bild der tabischen Hinterstrangdegeneration; daneben aber auch im Gehirn ein „umschriebener, graugelber mit Narbenstreifen in die Umgebung sich fortsetzender Herd am hinteren unteren und äusseren Ende des rechten Linsenkernes, $1 \mathrm{~cm}$ lang, $4 \mathrm{~mm}$ breit".

Audry ${ }^{2}$ ) beschreibt einen Fall von doppelseitiger Athetose bei Tabes bei einem 52 jährigen Mann. Der Verfasser betont dabei, dass die Bewegungsstörung ganz unabhängig von der Ataxie bestanden, dass der Kranke niemals cerebrale Attacken, niemals Hemiplegie oder dergl. gehabt habe. In seiner Arbeit stellt dann Audry noch mehrere Fälle zusammen, welche in das gleiche Gebiet gehören, aber choreiforme, oder auch epileptiforme Bewegungsstörungen darbieten, deren Betrachtung aber den Rahmen meiner Arbeit überschreiten würde.

Endlich konnte ich in der Literatur nur noch zwei Fälle dieser seltenen Combination beschrieben finden und zwar wurden diese von Laquer ${ }^{3}$ ) auf der XV. Wanderversammlung süddeutscher Neurologen und Irrenärzte mitgetheilt.

Der erste Fall stellte Athetosebewegungen bei einer 51jährigen, schon 8-10 Jahre an Tabes leidenden Kranken dar.

„Die krampfhaften, wurmförmigen, athetoiden Beuge- und Streckbewegungen erstreckten sich hier nur auf Fuss und Zehen, erschienen wie willkürliche, dauerten auch im Schlafe an."

Im zweiten Falle handelte es sich um einen 36 jührigen Mann, bei dem seit 5 Jahren deutliche Zeichen der Tabes aufgetreten waren. „Am Ende des vorigen Jahres (1889) begannen erst links, nach einigen Monaten auch rechts, die Tag und Nacht anhaltenden sonderbaren, fast grotesken, monotonen langsamen Bewegungen der Füsse und Zehen, die vom Willen des Patienten ganz unabhängig sind." Im

1) Rosenbach, Ist man berechtigt, den „Athetose“ genannten Symptomencomplex durch einen besonderen Namen auszuzeichnen? Virch. Arch. 68. 1876.

2) Audry, Des mouvements choréiformes et de l'athétose chez les ataxiques. Revue de médecine 1887.

3) Vgl. Neurolog. Centralblatt 1890. S. $380 / 81$. 
ersten Falle bestand von Seiten der Gehirnnerven Atrophie des Opticus, im zweiten Falle waren „Psyche, Sprache und Hirnnerven“ frei.

Ueber den Sitz des Leidens, der Athetose, sind die Meinungen getheilt, wie es auch bei den sog. primären Athetoseerkrankungen der Fall ist.

Die meisten Fälle von Athetose gehören der Kategorie der nach cerebralen Erkrankungen auftretenden Athetose an, und es sind hier auch wieder vorwiegend die cerebrale Kinderlähmung und ferner Apoplexien und Erweichungsherde, welche solche Bewegungsstörungen im Gefolge haben, und dieser Thatsache entsprechend finden wir unter den secundären Athetoseerkrankungen meist nur einseitige Bewegungsstörung mit mehr weniger vollständiger Hemiplegie vereinigt.

Der cerebrale Sitz ist bei diesen Erkrankungen ausser allem Zweifel, und nur die genauere Localisation ist keine einheitliche. Immerhin spricht aber die Ueberzahl der Sectionsbefunde dafür, dass bei der posthemiplegischen Athetose Krankheitsherde in der Nähe der grossen Basalganglien, ,in der überwiegenden Mehrzahl der Fälle im Seh- oder im Streifenhügel ${ }^{161}$ ) im Spiele sind.

Nach der Zusammenstellung von v. Monakow ${ }^{2}$ ) fanden sich wohl in 70-80 Proc. der zur Section gekommenen Fälle Läsionen im Gebiete der hinteren inneren Kapsel, der hinteren Sehhügelgegend oder der angrenzenden Abschnitte des Linsenkernes, in der Mehrzahl auf den Sehhügel beschränkt. Auch der Sitz der Erkrankung in der vorderen Vierhügelregion, in den Hirnschenkeln und im Pons ist beschrieben worden.

Das ziemlich häufige Eintreten von Athetose bei progressiver Paralyse hat zu der Discussion der Frage, ob auch eine Rindenläsion zu athetotischen Bewegungsstörungen führen könne, den Anlass gegeben. Dabei ist aber an die Thatsache zu erinnern, dass enge Beziehungen zwischen Hirnrinde und Basalganglien bestehen, wie dies v. Monakow durch den Nachweis der Degeneration der Thalamuskerne nach Rindenabtragung gezeigt hat. Es sind Fälle mitgetheilt, in denen nur Rindenveränderungen sich vorfanden, so Fälle von Balfour, Beach, Kurella u. A.; aber Eichhorst (l. o.) und auch L. Sander ${ }^{3}$ ) betonen dagegen, dass bei der progressiven Paralyse Gehirnläsionen nicht nur in der Gehirnrinde beobachtet werden, und dass die

1) Eichhorst, Ueber Athetose. Virch.'s Arch. Bd. 137. 1894.

2) v. Monakow, Gehirnpathologie. Nothnagel, Spec. Pathologie u. Therapie. TX. Bd. I. Thl. 1897.

3) Sander, Ein Fall von Athetose mit Sectionsbefund. Neurol. Centralblatt. 1897. S. 301 . 
Beobachtungen, auf Grund deren man die Möglichkeit der Hirnindenathetose annehmen zu müssen glaubte, nicht einwandsfrei sind.

Aber auch bei nicht mit Paralyse combinirter Athetose wollen viele Autoren die Hirnrinde verantwortlich machen. So fasst v. KrafftEbing einen Fall von doppelseitiger Athetose nach Erkältung als corticale motorische Rindenneurose auf, Koranyi führt die Athetose auf organische Veränderungen, chronische Entzündung, Sklerose in der Hirorinde zurück. Friedmann glaubt aus der Beeinflussung der Bewegungen durch Galvanisirung des Schädels bei einem Paralytiker auf den Rindensitz der Athetose schliessen zu dürfen.

Eulenburg ${ }^{1}$ ) spricht sich ebenfalls dafür ans, dass die Hirnrinde als Sitz der Athetose anzusehen ist, „dass der Ausgangspunkt der Athetosebewegungen nicht in subhemisphärischen basalen . Hirntheilen zu suchen sei, sondern in den motorischen Territorien der Hirnrinde".

Die Zahl der Fälle, in denen nur Hirnrindenherde bei Athetose gefunden wurden, ist zwar kleiner als die, bei denen Läsionen in der Gegend der Basalganglien bestanden; aber immerhin würde die Annahme, dass die Athetose auch von Hirnrindenherden ausgelöst werden könne, die Erklärung mancher Eigenheiten der Athetose erleichtern; so das doppelseitige Vorkommen, das oft vorkommende alleinige Befallensein der Hände, das Zusammenvorkommen mit Sprachstörungen und mit anscheinend corticaler Epilepsie. Endlich wären die Fälle, in denen keine Veränderungen im Gehirn gefunden wurden, eher verständlich, da wir gerade aus den neuesten Untersuchungen über nicht eitrige Encephalitis wissen, dass der Nachweis derartiger encephalitischer Herde der Rinde oft nur mit den subtilsten Methoden gelingt. Bestechend wäre es ausserdem auch gerade in unseren Fällen, bei der Combination der Tabes dorsalis mit Athetose an eine Läsion der Rinde zu denken, wenn wir die Jendrassik'sche ${ }^{2}$ ) Theorie der Localisation der Tabes berücksichtigen würden, welcher aus seinen Untersuchungen und Ueberlegungen die Schlussfolgerung zog, „dass der grösste Theil der tabischen Symptome aus der Rindenerkrankung abgeleitet werden muss, dass somit die Tabes dorsalis keine Rückenmarkskrankheit, sondern die des Gehirns ist", wofür aber der Beweis noch nicht erbracht ist.

Eisenloh $\mathbf{r}^{3}$ ) hat nun einen Fall publicirt, in dem trotz genaner

1) Eulenburg, Ueber Athetose. Wien. med. Presse 1889.

2) Jendrassik, Localisation der Tabes dorsalis. Deutsehes Arch. f. klin. Medicin. 1888. Bd. 43.

3) Eisenlohr, Zur pathologischen Anatomie der Athetose. Jahrb. der Hamburg. Staatskrankenanstalten. 1896. Bd. IV; vgl. Neurolog. Centralbl. 1896. 
Untersuchung keine Veränderungen im Gehirn gefunden werden konnten, in dem aber wohl sehr erhebliche Iüsionen des Rückenmarks nachweisbar waren. Er fasst die Veränderungen als chronische centrale Myelitis, analog etwa der multiplen Sklerose, auf, welche möglicherweise congenital entstanden ist.

Eis enlohr betont aber, dass es auffallend war, dass jede Muskelatrophie und ferner alle Veränderungen fehlten, welche die Betheiligung der mimischen und Sprachmuskeln erklären.

Dass das Fehlen klinischer Gehirnsymptome nicht gegen die Localisation der Athetose im Gehirn spricht, das beweisen die zahlreichen Fälle, in denen bei anscheinend idiopathischer, sowohl einseitiger wie doppelseitiger Athetose, bei der Obduction Gehirnherde nachgewiesen werden konnten.

Bei den Fällen von Combination einer Rückenmarkserkrankung mit Athetose, welche nicht zur Obduction gelangt sind, ist es deshalb ein müssiger Streit, ob die Athetosebewegungen vom Gehirn allein oder auch rom Rückenmark aus erzeugt werden.

Ein der Athetose nahe verwandtes Krankheitsbild, das der chronischen Chorea, ist bei Syringomyelie von Duchenne und Hoffmann ${ }^{1}$ ) beschrieben worden, doch fassen die Autoren dies Zusammentreffen nur als rein zufälliges auf, als eine Combination verschiedenartiger Krankheitsprocesse, besonders da im Falle von Hoffmann ausser den bereits erwähnten Processen noch Epilepsie und Poliomyelitis anterior chronica bestand.

Die bei Syringomyelie vielfach beobachteten choreiformen Charakter aufweisenden Spontanbewegungen sind wohl nicht mit echter Chorea zu identificiren, ihr Ursprungsort kann das Rückenmark sein.

Aber nicht nur an das Rückenmark als Sitz der Athetose und Chorea ist gedacht worden, sondern auch die peripheren Nerven wurden zur Localisation des Processes herangezogen. Löwenfeld ${ }^{2}$ ) hat bei multipler Neuritis Bewegungen in den befallenen Extremitäten beobachtet, welche er ohne Bedenken als Athetosis bezeichnen zu können glaubt. Die sich in Folge der Kritik dieser Bezeichnung durch Remak ${ }^{2}$ ) anschliessende Discussion hat keine endgültige Bestätigung der Richtigkeit der Bezeichnung gegeben, lässt vielmehr darauf schliessen, dass

1) Hoffmann, Zur Lehre von der Syringomyelie. Deutsche Zeitschrift f. Nervenheilkunde. Bd.'III, und Schlesinger, Die Syringomyelie. Wien 1895.

2) Löwenfeld, Ein Fall von multipler Neuritis mit Athetosis. - Remak, Ein Fall von generalisirter Neuritis mit schweren elektrischen Alterationen auch der niemals gelähmten Nn. faciales. Repliken von Löwenfeld und Remak, Neurologisches Centralblatt. 1885. 
die Anwendung des Namens Athetose auf die Bewegungsstörung nicht ganz berechtigt war.

Aehnlich unklare Bezeichnungen der in Frage kommenden Bewegungsstörungen bei zwei Fällen von Pseudotabes, welche Rossolimo ${ }^{1}$ ) bespricht, lassen eine Verwerthung im Sinne der Localisation der Bewegungsstörung in den peripheren Nerven nicht zu.

Eine interessante Complication von myotonischen Störungen mit Athetose theilt Kaiser ${ }^{2}$ ) mit.

Der Kranke bot das typische Bild der Athetose und das typische Bild der Myotonie, nur war keine hereditäre Belastung nachzuweisen und fehlte die elektrische myotonische Reaction. Der Verf. kommt denn auch zu dem Schlusse, dass die myotonischen Störungen nur die äussere Form, nicht die pathologisch-anatomische Ursache mit der familiären, echten Myotonie gemein haben, und dass die Erkrankung in das cortico-motorische Neuron zu localisiren sei und zwar in den centralen Endpunkt dieses Neurons, die Ganglienzellen des Grosshirns, auf welche nach $\mathrm{Kais}$ er die gleichzeitig bestehende Hypertrophie der rechten Körperseite noch besonders hinweise.

Die Mehrzahl der Autoren, welche die Combination ron Tabes mit Athetose beobachtet haben, geben ihre Ansicht dahin ab, dass die Athetose auch spinal entstehen könne, so Audry, Laquer, Rosenbach.

Auffallend ist es aber, dass gerade in dem einzigen derartigen Falle, welcher zur Section kam, sich auch ein Gehiruherd und zwar gerade in der Gegend der Basalganglien vorfand.

In den von mir beschriebenen Fällen liessen sich keine anamnestische Daten für eine etwaige Gehirnläsion ermitteln, und es bestanden auch keine Herdsymptome, höchstens liesse im zweiten Falle die zeitweise aufgetretene Abducensparese und der Nystagmus an eine Betheiligung des Gehirns denken. Auch von progressiver Paralyse bestanden in unseren Fällen keine Symptome, obwohl ja bei der Häufigkeit der Combination von Tabes mit progressiver Paralyse und den zahlreichen Beobachtungen des Vorkommens von Athetose bei der Paralyse gerade an das Bestehen einer Taboparalyse in erster Linie gedacht werden musste.

Wir können aber auch jedenfalls das Bestehen einer Gehirnläsion nicht ausschliessen, da wir wissen, dass nicht allzu selten bei Obduc-

1) Rossolimo, Zur Pathogenese der unwillkürlichen Zuckungen bei Tabes. Gesellschaft der Neuropath. und Irrenärzte zu Moskau. 30. IV. 1893. Neurol. Centralblatt 1893. S. 839.

2) Kaiser, Myotonische Störungen bei Athetose. Neurolog. Centralbl. 1897. 
tionen latente Hirnherde gefunden werden, und da wir bei dem Zusammenhang der Tabes mit Syphilis und der häufigen Combination der Tabes mit den auf gleicher ätiologischer Grundlage entstandenen Arterienveränderungen oder Meningealveränderungen der völligen Intactheit des Gehirns niemals sicher sind.

Auch die klinischen Erscheinungen der Athetose sprechen eher für deren Localisation im Gehirn.

Gerade die Zusammenstellung dieser Bewegungsstörung mit den Zuckungen, Krämpfen und Contracturen bei Tabes, wie sie Audry macht, fordert unseren Widerspruch heraus.

Audry bespricht die athetotischen Bewegungen bei Tabes im Zusammenhange mit den choreiformen Bewegungen, den Zuckungen, Krämpfen und Contracturen und kommt zu dem Schlusse, dass die athetotischen und choreiformen Bewegungen nur eine Steigerung der Zuckungen sind und alle diese Bewegungsstörungen nur der Stärke nach, nicht der Art nach verschieden seien.

Er glaubt, dass alle diese Bewegungen von einer accessorischen Iuäsion der motorischen Seitenstränge herrührten, dass die Fälle also alle zur Gruppe der Tabes combiné (wie sie Grasset) zusammenfassend charakterisirt hat) gehörten. Die Bedenken, welche sich aus dem Mangel jeder Läsion der Seitenstränge in den Obductionsbefunden von Rosenbach und Cruveilhier ergeben, glaubt er zerstreuen zu können, indem er zu erwägen giebt, dass die Läsion der Seitenstränge dem blossen Auge meist nicht sichtbar sind, dass ferner, wenigstens im Falle von Cruveilhier, bei dem es sich um die Combination von Chorea mit Tabes handelte, eine mikroskopische Untersuchung nicht gemacht worden ist, und dass Rosenbach das Fehlen jeder Läsion der Seitenstränge nicht besonders betont hat.

Gegen diese Zusammenstellung der verschiedenen bei Tabes vorkommenden Bewegungen spricht aber das klinische Bild.

Die athetotischen und choreiformen Bewegungen sind so complicirte und wohlcharakterisirte Bewegungen, dass sie klinisch von den zeitlich wie örtlich ganz unregelmässigen Zuckungen spinaler Natur absolut scharf zu trennen sind.

Eher zu vergleichen, freilich nicht im klinischen Bild, sondern in ihrer Regelmässigkeit, sind die Athetose und Chorea mit der Paralysis agitans, deren Ursprung mit grösster Wahrscheinlichkeit auch in das Gehirn zu verlegen ist; auch Combinationen der Paralysis

1) Grasset, Du tabes combiné (ataxo-spasmodique) ou sclérose postérolatérale de la moëlle. Archiv de neurol. 1886. XI/XII. 
398 XXI. Artsperger, Ueber Athetose als Complication von Tabes dorsalis.

agitans mit Tabes sind ja bereits beobachtet und z. B. von Hess ${ }^{1}$ ) und Placzek²) veröffentlicht worden.

Der directe Beweis für die unbedingte cerebrale Entstehung der die Tabes dorsalis complicirenden Athetose ist freilich noch nicht erbracht und wird nur durch den Befund am Sectionstisch erbracht werden können, aber die Wahrscheinlichkeit spricht meiner Ansicht nach dafür, dass auch in dem Falle der Combination der Athetose mit Räckenmarkserkrankungen Gehirnveränderungen vorhanden sind.

1) Hess, Paralysis agitans mit Tabes. Aerztl. Verein Hamburg. 24. IV. 1900. Ref. Therapeut. Monatshefte. 1900. Heft 6.

2) Placzek, Ueber das Zusammenvorkommen von Paralysis agitans und Tabes dorsalis. Sitzg. der Berl. Gesellsch. f. Psychiatr. und Nervenkrankh. vom 14. Dec. 1891. 